\title{
Multiple Emulsions Able to Be Used for Oral Administration of Active Pharmaceutical Ingredients: Physico-Chemical Parameters Study of Different Phases
}

\author{
Louis Augustin Diaga Diouf', Alphonse Rodrigue Djiboune', Mamadou Soumboundou², \\ Sidy Mouhamed Dieng ${ }^{3}$, Papa Mady Sy¹, Gora Mbaye¹, Mounibé Diarra ${ }^{1}$ \\ ${ }^{1}$ Pharmaceutical Physics and Biophysics Laboratory, Faculty of Medicine, Pharmacy and Odontology, Cheikh Anta Diop \\ University of Dakar, Dakar, Senegal \\ ${ }^{2}$ Pharmaceutical Physics and Biophysics Laboratory, Healthy UFR of Thies, University of Thies, Thies, Senegal \\ ${ }^{3}$ Galenic and Industrial Pharmacy Laboratory, Healthy UFR of Thies, University of Thies, Thies, Senegal \\ Email: louyoguiste@yahoo.fr, alphonserodrigue.djiboune@ucad.edu.sn, mamadou.soumboundou@univ-thies.sn, \\ sidym.dieng@univ-thies.sn,papamady.sy@ucad.edu.sn,Gora.mbaye@ucad.edu.sn,mounibe.diarra@ucad.edu.sn
}

How to cite this paper: Diouf, L.A.D., Djiboune, A.R., Soumboundou, M., Dieng, S.M., Sy, P.M., Mbaye, G. and Diarra, M. (2020) Multiple Emulsions Able to Be Used for Oral Administration of Active Pharmaceutical Ingredients: Physico-Chemical Parameters Study of Different Phases. Journal of Modern Physics, 11, 1528-1535. https://doi.org/10.4236/jmp.2020.1110094

Received: July 15, 2020

Accepted: October 11, 2020

Published: October 14, 2020

Copyright $\odot 2020$ by author(s) and Scientific Research Publishing Inc. This work is licensed under the Creative Commons Attribution International License (CC BY 4.0).

http://creativecommons.org/licenses/by/4.0/

\section{(c) (i) Open Access}

\begin{abstract}
Multiple emulsions are of great therapeutic interest especially in the administration of medicines which can be inactivated by digestive enzymes; moreover the researches of formulation not being often easy, a control of the different phases physicochemical parameters would be of great interest in rapid formulations and at low cost. When formulating emulsions, the preliminary tests, also known as formulation tests, constitute a step which can be long and expensive because of the quantity of reagents that can be used. A rigorous methodology could thus be of great interest, which is at the aim of our study which consists of evaluating the physico-chemical parameters of different phases used to make thus multiple emulsions. In our study, physico-chemical parameters such as conductivity, $\mathrm{pH}$, density, viscosity, and surface tension have been studied by direct measurement using equipment and also by means of suitable mounting. The results showed that the $\mathrm{pH}$ and the surface tension have an important role in the prediction of the stability of emulsions, these latter must be of the same order of magnitude. For all phases conductivity does not have too much interest apart from helping to determine the type of the emulsion.
\end{abstract}

\section{Keywords}

Active Pharmaceutical Ingredients, Multiple Emulsions, Stability, 
Physico-Chemical Parameters

\section{Introduction}

Emulsions are thermodynamically unstable systems; they are mixtures of two immiscible phases to be dispersed one within the other. The main goal is to keep this dispersion stable for a long time. In practice, according to Salager, "formulators of emulsions have experienced the unpleasant occurrence of the lack of reproduction of the physical properties (type, stability, viscosity) of an emulsion formulated with identical raw materials following the same rigorously definitive experimental protocol" [1] [2] [3]. Based on this assertion, we have studied the physical parameters which allow us to find information that is relevant for obtaining emulsions with good stability and which would be carried out within a rational time.

With regard to emulsions in general, many studies have been carried out on the various techniques which improve their quality and stability. These techniques are based on compositional, formulation and process variables [4] [5] [6] [7]. Concerning the formulation and composition variables, the type of surfactant, the HLB, and also the proportions of the various constituents were studied.

\section{Material}

\subsection{Reagents}

For the formulation of multiple emulsion, a mixture of $\operatorname{Span}^{\circledast} 80 /$ Tween $^{\circledast} 80$ which HLB can vary from 4.3 to 15 is used as surfactant for oil in water emulsion, the surfactant used for water in oil emulsion is Montane $e^{\circledast} 481 \mathrm{VG}$ (M481VG) (HLB 4.5).

The oily or lipophilic phase used was peanut oil and the hydrophilic one consisted of Phosphate buffer Saline (PBS) pH 7.2; mixed with Carboxymethylcellulose (CMC).

\subsection{Equipment}

Equipment used consisted of:

- $\mathrm{pH}$ meter Schott Geräte CG820

- Conductimeter Schott Geräte CG820

- Magnetic stirrer fisher scientific

- Precision balance Ohaus explorer

- Surface tension meter Dognon-Abribat model PROLABO

\section{Methods}

For both the lipophilic and the hydrophilic phase, the physico-chemical parameters as $\mathrm{pH}$, conductivity, surface tension and viscosity are studied through the above cited apparatus. To measure the viscosity, the method used consisted to 
form a drop of dispersed phase in the dispersing phase and in measuring the speed of migration of the drop thus formed, the device is shown in Figure 1.

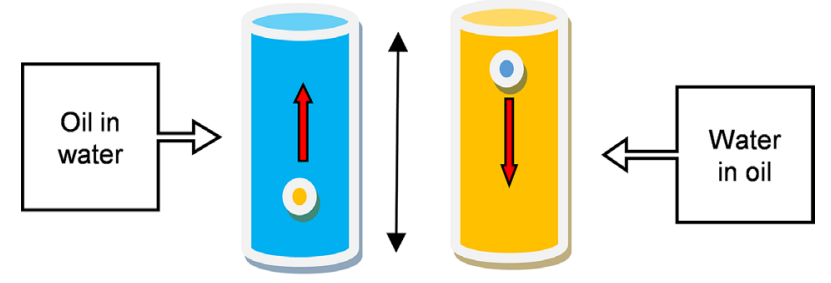

Figure 1. Viscosity measuring device [6].

The following Equation (1), determines the viscosity obtained using the limiting velocity reached by a moving particle in a viscous medium:

$$
\eta=\frac{2\left(\rho_{\text {drop }}-\rho_{\text {medium }}\right)}{9 \cdot v_{\text {limit }}} \cdot g \cdot r_{\text {drop }}^{2}
$$

$\eta=$ viscosity, $\rho=$ density, $v=$ velocity of the formed drop, $r=$ radius of the formed drop, $g=$ gravity acceleration.

It was also studied the changes of the contact angles by observing the behavior of two superposed and not mixed phases [8].

The hydrophilic phase was mixed with $1 \%$ of CMC and with the emulsifiers constituted by the span $80 /$ tween 80 pair at $10 \%$. The proportion of the mixture of $\operatorname{Span}^{\oplus} 80 /$ Tween $^{\oplus} 80$ which gave different HLB is indicated in Table 1.

Table 1. Proportions of span 80/tween 80 emulsion for HLB ranging from 7 to 14 .

\begin{tabular}{ccc}
\hline Span 80 & Tween 80 & HLB \\
\hline $74.77 \%$ & $25.23 \%$ & 7 \\
$65.42 \%$ & $34.58 \%$ & 8 \\
$46.73 \%$ & $53.27 \%$ & \multicolumn{2}{c}{10} \\
$28.04 \%$ & $71.96 \%$ & \multicolumn{2}{c}{12} \\
$9.35 \%$ & $90.65 \%$ & \multicolumn{2}{c}{14}
\end{tabular}

The HLBs vary from 7 to 14 and are intended for the production of $\mathrm{O} / \mathrm{W}$ emulsions.

Concerning the surfactant Montane ${ }^{\circledast} 481$ VG (M481VG) used to prepare W/O emulsion his HLB is fixe (4.5) and the proportion used is noted in Table 2.

Table 2. Proportions of M481VG used for W/O emulsion.

\begin{tabular}{cc}
\hline Sample & Composition (\%) \\
\hline M1 & 5 \\
M2 & 6 \\
M3 & 7 \\
M4 & 8 \\
M5 & 10 \\
\hline
\end{tabular}




\section{Results}

\subsection{Hydrophilic Phase}

The hydrophilic phase consists essentially of $1 \%$ CMC solution for which the $\mathrm{pH}$ measurement gave a value of 6.8 , therefore slightly acidic. For the conductivity, its measurement gave a value of $17 \mathrm{mS} / \mathrm{cm}$ for the $1 \% \mathrm{CMC}$ solution and 18 $\mathrm{mS} / \mathrm{cm}$ for the $2 \%$ solution.

The surface tension determined by the immersion blade method gave a value of $38.50 \mathrm{mN} / \mathrm{m}$.

For the hydrophilic phase with $1 \%$ CMC mixed with the emulsifiers constituted of the span $80 /$ tween 80 pair at $10 \%$, the results of the measurements of the $\mathrm{pH}$, conductivity and surface tension are listed in Table 3.

Table 3. Physico-chemical parameters of the CMC solution containing $10 \%$ span 80/tween 80 mixture.

\begin{tabular}{cccc}
\hline HLB & $\mathrm{pH}$ & Conductivity $(\mathrm{mS} / \mathrm{cm})$ & Surf. Tens. $(\mathrm{mN} / \mathrm{m})$ \\
\hline 7 & 5.25 & 0.91 & 30.656 \\
8 & 5.13 & 0.96 & 31.392 \\
10 & 4.9 & 1.01 & 29.675 \\
12 & 4.81 & 1.02 & 30.961 \\
14 & 4.66 & 1.15 & 31.147 \\
\hline
\end{tabular}

It is noted a decrease in $\mathrm{pH}$ corresponding to an increase in the degree of acidity, a slight increase in conductivity and small fluctuations in the surface tension between 29 and $31 \mathrm{mN} / \mathrm{m}$.

\subsection{Lipophilic Phase}

The results of $\mathrm{pH}$ measurements of the lipophilic phase, in which the proportions of emulsifying agent vary from $0 \%$ to $8 \%$, are shown in Table 4 .

Table 4. $\mathrm{pH}$ measurements of the lipophilic phase.

\begin{tabular}{cc}
\hline Proportions & $\mathrm{pH}$ \\
\hline $0 \%$ & 2.78 \\
$4 \%$ & 3.8 \\
$6 \%$ & 3.5 \\
$8 \%$ & 4.3 \\
\hline
\end{tabular}

An acidic $\mathrm{pH}$ is observed for all lipophilic phases, we noted an increase with the proportions of surface-active agent constituted by M481VG.

For different concentrations of montane $481 \mathrm{VG}$ we also measured the surface tension which results are shown in Figure 2. 


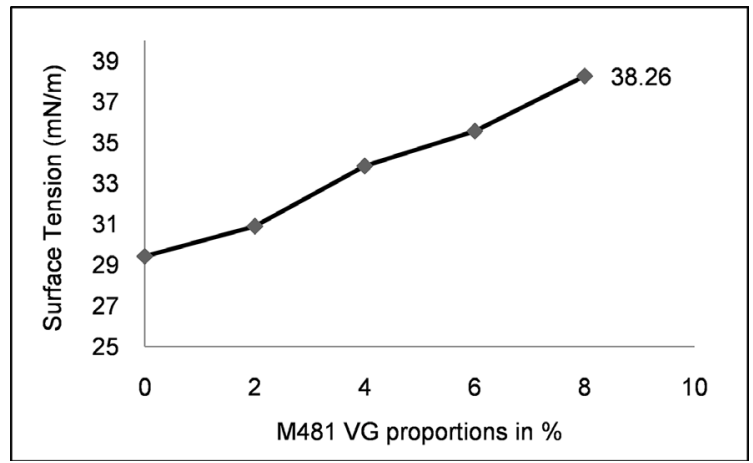

Figure 2. The surface tension variation curve of lipophilic phase as a function of montane 481 VG concentration.

These results show that montane 481 VG increases surface tension instead of decreasing it, this is justified in so far as we have emulsions in which it is the most dense phase that must be introduced in the least dense phase, this explains the need to increase the density of the latter to avoid the sedimentation of the internal phase due to the action of gravity [8] [9].

These changes in interfacial tension are also visible when the two solutions are superposed. A decrease in the contact angle of the oil phase with the wall of the beaker is thus observed as shown in Figure 3 following the Equation (2)

$$
F=2 \pi R s \cdot \cos \theta
$$

$s=$ surface tension coefficient, $R=$ droplet radius, $q=$ contact angle.

There is also a beginning of penetration of the oil phase into the aqueous one, which indicates a decrease in the interfacial tension.
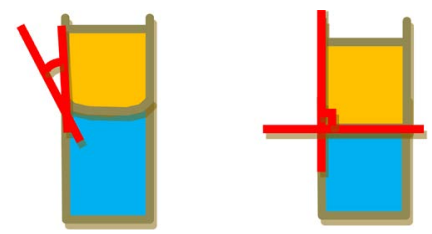

Figure 3. Variation of the contact angle of the lipophilic phase before $\left(16^{\circ}\right)$ and after $\left(90^{\circ}\right)$ addition of the surfactant [8].

For the measurement of the density, the results are given in Figure 4.

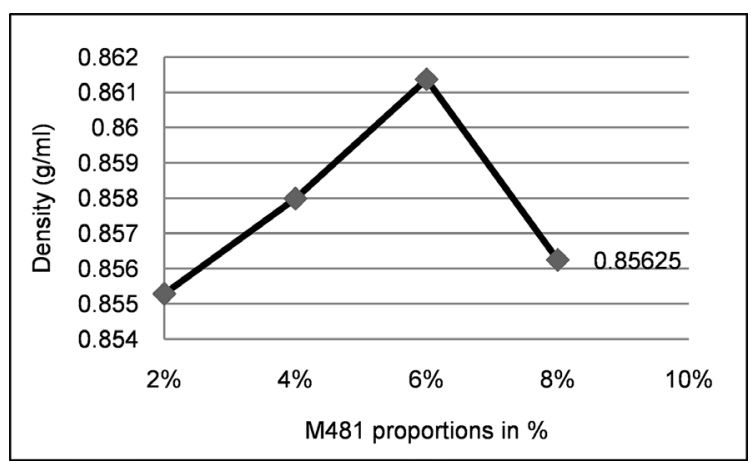

Figure 4. Variation of the density as function of concentration of montane $481 \mathrm{VG}$. 
A maximal density is noted at the concentration of $6 \%$ of M481VG in the lipophilic phase.

The results of the viscosity measurements, obtained by the immersion drop method identical to the ball drop method, are shown in Figure 5.

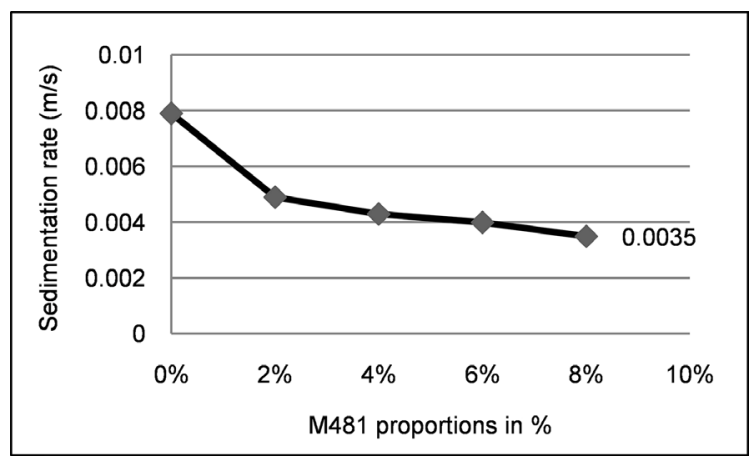

Figure 5. Variation of the sedimentation rate as a function of montane $481 \mathrm{VG}$ concentration.

\section{Discussion}

The emulsions intended to do are W/O/W emulsions type [8] [9]. For the realization we used three types of surfactants that allowed varying the hydrophilic/lipophilic balance. These surfactants are, tween 80 and span 80, but also montane 481VG.

Data from the literature have shown that emulsifiers for water-in-oil emulsions must have an HLB between 1 and 6, hence the use of the montane 481VG which has HLB equal to 4.5, and for oil-in-water emulsions the HLB must be between 7 and 14 hence the use of span 80 tween 80 couple [3] [10].

The study of the physico-chemical parameters such as $\mathrm{pH}$, conductivity and surface tension allowed seeing the variations of these properties as function of HLB especially with regard to the external hydrophilic phase. Since the goal was the formulation of multiple emulsions, the stability of the latter depends more on the external aqueous phase. For the latter, composition was of the utmost importance. Thus, as for the internal aqueous phase for which the $\mathrm{pH}$ is slightly acid in order of 6.8 , the acidic $\mathrm{pH}$ is also observed for the external aqueous phase, which decreases as the HLB increases. The $\mathrm{pH}$ of the external hydrophilic phase to which we obtained a stable emulsion (HLB 8) of the order of 5.13 is quite close to that of the lipophilic phase with $6 \%$ emulsifier having a $\mathrm{pH}$ of 4.3 . This acidity of the $\mathrm{pH}$ of the two phases is a stability indicator because an identical zeta potential can be observed around the droplets, which may be at the origin of a force of electrostatic repulsions between the droplets [11] [12] [13].

We also noted that the HLB of span/tween 80 torque giving stable emulsions are the same as those found by ANKURMAN [14].

The most interesting parameter we have studied is the surface tension at the interface of two liquids. Indeed, the main objective in the formulation of the emulsions is to reduce the interfacial tension. Therefore, if the surface tension of 
the aqueous phase oscillates between 29 and $30 \mathrm{mN} / \mathrm{m}$, that of the oily phase increases in proportion to the M481VG concentration and varies from 29 to 38 $\mathrm{mN} / \mathrm{m}$. Thus, superficial tensions observed are practically the same order of magnitude. It is also noted that the surface tension of the aqueous phase has decreased by almost half. We have tried to materialize that drop in the interfacial tension by measuring the contact angle of the aqueous phase. This shows an increase of the contact angle which has passed from $16^{\circ}$ before addition of the surfactant to $90^{\circ}$ after addition of $6 \%$ of surfactant. This makes the analysis of the evolution of the surface tension of two liquids a good indicator in the prediction of stability [15]. Besides $\mathrm{pH}$ and interfacial tension, the viscosity measurement can also give information about the feasibility of the emulsions. Indeed, by observing the curve of variation of the viscosity by means of the sedimentation rate of a drop of dispersed phase, it is observed that this velocity decreases and tends to stabilize around a value which, in regard to our emulsions, is $6 \%$.

\section{Conclusions}

The study of the physico-chemical parameters such as $\mathrm{pH}$, conductivity, surface tension... allowed seeing the variations of these properties as function of HLB especially with regard to the external hydrophilic phase. Since the goal was the formulation of multiple emulsions, the stability of the latter depends more on the external aqueous phase. For the latter, composition was of the utmost importance.

When making emulsions, the preliminary tests, also known as formulation tests, constitute a step which can be long and expensive because of the quantity of reagents that can be used. A good methodology could therefore be of great interest. Thus the results of the study showed that the $\mathrm{pH}$ and the interfacial tension have an important role in the prediction of stability of emulsion; the interfacial tension of the two phases must be of the same order of magnitude. The measurement of the conductivity does not have too much interest apart from helping to determine the type of the emulsion.

\section{Author Contributions}

All authors have contributed to the research and design of this item.

\section{Conflicts of Interest}

The authors declare no conflicts of interest regarding the publication of this paper.

\section{References}

[1] Salager, J.L., Anton, R. and Aubry, J.M. (2006) Formulation des émulsions par la méthode HLD. In Techniques de l'ingénieur, vol génie des procédés J2, Chapter 158, 1-16.

[2] Becher, P. (1983-96) Encyclopedia of Emulsion Technology. Vol. 4, M. Dekker, New York. 
[3] Griffin, C. (1949) Journal of Cosmetic Science, 1, 311-326.

[4] ICI Americas Inc. Wilmington (1984) The HLB System, a Time Saving Guide to Emulsifier Selection.

[5] Silva Cunha, A., Grossiord, J.L., Puissieux, F. and Seiller, M. (1997) International Journal of Pharmaceutics, 158, 79-89. https://doi.org/10.1016/S0378-5173(97)00249-4

[6] Kaci, M., Meziani, S., Arab-Tehrany, E., Gillet, G., Desjardins-Lavisse, I. and Desobry, S. (2014) Ultrasonics Sonochemistry, 21, 1010-1017. https://doi.org/10.1016/j.ultsonch.2013.11.006

[7] Li, J., Su, L., Li, J., Liu, M.-F., Chen, S.-F., Li, B., Zhang, Z.-W. and Liu, Y.-Y. (2015) RSC Advances, 5, 83089-83095. https://doi.org/10.1039/C5RA11155B

[8] Diouf, L.A.D. (2017) Elaboration et évaluation d'émulsions multiples pour l'administration de l'insuline par voie orale. Thèse de doctorat de PhysiqueBiophysique 2017 Université Cheikh Anta DIOP Dakar (Sénégal).

[9] Diouf, L.A.D., Mbaye, G., Ndiaye, A., Sy, P.M., Djiboune, A.R., et al. (2015) International Journal of Current Research, 7, 22420-22423.

[10] Lange, N.A. and Dean, J.A. (1967) Lange's Handbook of Chemistry. 10th Edition, McGraw Hill, New York, 1661-1665.

[11] Gadhave, A. (2014) International Journal of Science and Research, 3, 573-575.

[12] Emeche, A.K., Okujagu, D.C. and Okwu, E. (2017) International Journal of Research and Development, 2, 1-8.

[13] Strassner, J.E. (1968) Journal of Petroleum Technology, 20, 303-312. https://doi.org/10.2118/1939-PA

[14] Shrestha, A. (2011) Effect of Span 80-Tween 80 Mixture Compositions on the Stability of Sunflower Oil-Based Emulsions Bachelor of Technology in Biotechnology Rourkela India.

[15] Kanouni, M., Rosano, H.L. and Naouli, N. (2002) Advances in Colloid and Interface Science, 99, 229-254. https://doi.org/10.1016/S0001-8686(02)00079-9 